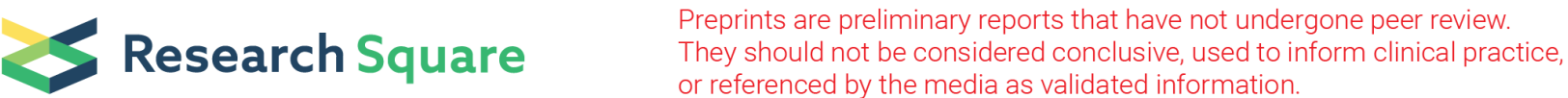

\section{Multi-Objective Casting Production Scheduling Problem by a Neighborhood Structure Enhanced Discrete NSGA-II: An Application from Real-World Workshop}

\author{
Weihua Tan ( $\nabla$ tanwh@hnu.edu.cn ) \\ Hunan University https://orcid.org/0000-0003-0920-1522 \\ Xiaofang Yuan \\ Hunan University https://orcid.org/0000-0001-7280-7207 \\ Yuhui Yang \\ Hunan University \\ Lianghong Wu \\ Hunan University of Science and Technology
}

\section{Research Article}

Keywords: Multi-objective casting production scheduling problem, Defective rate, Variable processing speed, Non-dominated sorting genetic algorithm, Neighborhood structure

Posted Date: November 1st, 2021

DOI: https://doi.org/10.21203/rs.3.rs-1006864/v1

License: (c) (i) This work is licensed under a Creative Commons Attribution 4.0 International License.

Read Full License 


\title{
Multi-objective casting production scheduling problem by a neighborhood structure enhanced discrete NSGA-II: an application from real-world workshop
}

\author{
Weihua Tan ${ }^{1}$. Xiaofang Yuan ${ }^{1, *}$. Yuhui Yang ${ }^{1}$. Lianghong $\mathrm{Wu}^{2}$ \\ Received: date / Accepted: date
}

\begin{abstract}
Casting production scheduling problem (CPSP) has attracted increasing research attention in recent years to facilitate the profits, efficiency, and environment issues of casting industry. Casting is often characterized by the properties of intensive energy consumption and complex process routes, which motivate the in-depth investigation on construction of practical multiobjective scheduling models and development of effective algorithms. In this paper, for the first time, the multi-objective casting 10 production scheduling problem (MOCPSP) is constructed to simultaneously minimize objectives of defective rate, makespan, and total energy consumption. Moreover, a neighborhood structure enhanced discrete NSGA-II (N-NSGA-II) is designed to better cope with the proposed MOCPSP. In the N-NSGA-II, the advantage of selection mechanism of NSGA-II is fully utilized for selecting non-dominate solution, three neighborhood structures are elaborately designed to strengthen the ability of the local search, and a novel solution generating approach is proposed to increase the diversity of solutions for global search. Finally, 15 a real-world case is illustrated to evaluate the performance of the N-NSGA-II. Computational results show that the proposed N-NSGA-II obtains a wider range of non-dominated solutions with better quality compared to other well-known multi-objective algorithms.
\end{abstract}

Keywords Multi-objective casting production scheduling problem $\cdot$ Defective rate $\cdot$ Variable processing speed $\cdot$ Non-dominated sorting genetic algorithm $\cdot$ Neighborhood structure

\section{Introduction}

Casting is one of machine manufacture industry foundation crafts, which is closely tied with the development of equipment manufacturing industries like automobile, machine tool, aviation, aerospace, and national defense. In the modern casting industry, multi-variety and small batch production mode is becoming popular and necessary [6] in order to meet the growing demand for customization products. Meanwhile, due to the energy-intensive [14] nature of casting industry, efficient and green casting manufacturing earns an increasing concern for the last decades [28]. As we known, efficient scheduling can serves as a powerful tool to improve the production cost, manufacturing cycle and environment issue [30]. Therefore, the casting production scheduling problem (CPSP) under the shop floor environment for practical application attracts increasing attention from both the industry and the research community.

Two main specific attributes of casting process need to be considered when dealing with CPSP. Firstly, casting processing is a typical energy-intensive and high-pollution manufacturing [36]. Some operations such as melting and thermal treatments will involve great amount of time, energy consumption and pollute emission. Therefore, multi objectives need to be simultaneously addressed in CPSP. Secondly, the casting process is extremely complex [30]. On the one hand, the casting products generally have long process routes and the routes are various for different casting products in the mixed production lines. On the other hand, large number of multi-functional machines are involved in the casting workshops. These characteristics lead to enormous feasible scheduling schemes of CPSP, necessitating the development of powerful optimization algorithms.

The CPSP can be viewed as the extension of flexible job-shop scheduling problem(FJSP) [30]. In pioneer works, objectives of makespan and total energy consumption are widely considered in FJSP [37]. Mokhtari et.al [25] develop joint scheduling of the production and the maintenance operation in the flexible job shop environment with the objectives to minimize the makespan, total energy consumption and maximize the total availability of system. Wu et.al [40] consider deterioration effect into FJSP, in the actual processing time of the operation is variable under the influence of the deterioration effect, and the FJSP-DEEC model is proposed to optimize the makespan and the total energy consumption. Moreover, Dai et.al [8] address an energyefficient flexible job shop scheduling problem with transportation constraint and formulate a multi-objective optimization model to minimize the objectives of makespan and the total energy consumption. As demonstrated above, the performance of the

$\triangle$ Xiaofang Yuan

E-mail: yuanxiaofang@hnu.edu.cn

1 College of Electrical and Information Engineering, Hunan University, Changsha, Hunan, 410082, China

2 College of Information and Electrical Engineering, Hunan University of Science and Technology, Xiangtan 411201, China 
shop floor has been significantly promoted by optimizing makespan and the total energy consumption. Besides, defective rate is another vital issue for casting production. In the practical workshop, the performance of machine tool deteriorates gradually with the production process [42], resulting in the increase in defective rate. Due to the attributes of casting process such as long operation-chain, low defective rate of each operation in the route is stringent required. Moreover, because of the energy-intensive property of casting, wide-employed maintenance strategies [35,31] which require frequent shutting down of machines is costly and insufficient for controlling the defective rate of casting products. Unfortunately, to the best of our knowledge, no existing 50 research has addressed the objective of defective rate in CPSP. To this end, a multi-objective casting production scheduling problem (MOCPSP) is constructed considering the objectives of defective rate, makespan, and total energy consumption simultaneously in this paper.

The proposed MOCPSP is an NP-hard problem. With the nature of extreme complexity, exact approach is unable to find optimal solutions in a reasonable time when the problem size is large [11]. Various multi-objective heuristic techniques have 55 been proposed in the last decades. Recent researches [4]12] employ different hybrid algorithms to solve multi-objective FJSP by combining some of those heuristic techniques and achieved remarkable results. Shen et.al [33] develop a modified multiobjective evolutionary algorithm to solve the MOFJSP. Zhang et.al [45] propose a hybrid optimization algorithm for MOFJSP combining a particle swarm optimization algorithm and a tabu search algorithm. Li et.al [18, 19] develop two hybrid algorithms by integrating tabu search algorithm with variable neighborhood search strategy. Moslehi et.al [26] report a hybrid particle swarm 60 optimization with a special designed local search strategy. Yuan et.al [43] propose new memetic algorithms by incorporating a novel local search algorithm into the NSGA-II. Shao et.al [32] develop a hybrid discrete particle swarm optimization combined with simulated annealing algorithm to solve the MOFJSP with objectives of minimizing makespan, maximal machine workload and total workload of machines. To better cope with the proposed MOCPSP, an efficient hybrid heuristic algorithm with enhanced performance is designed in this paper.

65 The main contributions of this paper can be outlined as follows:

(i) To narrow the gap between theoretical research and real-world casting production scheduling application, a novel MOCPSP model is developed considering defective rate, makespan and total energy consumption simultaneously, which is the first study in this area.

(ii) Facing the extreme complexity of the proposed MOCPSP, a neighborhood structure enhanced discrete NSGA-II (NNSGA-II) is developed. In the N-NSGA-II, three neighborhood structures are elaborated designed to strengthen the ability of the local search and a novel solution generating approach is proposed to increase the diversity of solution for global search.

The remainder of this paper is organized as follows. Section 2 describes the MOCPSP in detail and gives the formulation of the MOCPSP. Section 3 illustrates the proposed N-NSGA-II algorithm for solving the MOCPSP. Section 4 presents the computational results and analysis of a real-world casting case by the N-NSGA-II and other well-known algorithms. The conclusion and future work are presented in Section 5 .

\section{Description and formulation of the MOCPSP}

In this section, the overall description of the proposed MOCPSP is given firstly. After that, the models of the three objectives are formulated respectively. The formulation of the MOCPSP model is presented at last.

\subsection{Problem description}

In the MOCPSP, the objectives of defective rate, makespan, and total energy consumption are addressed as the indicators of production quality, efficiency, and environmental issues. In most practical workshop, the processing speed of machine is changeable [3]. For example, the processing speed of computer numerically controlled manufacturing machine for an operation is adjusted by controlling cutting speed or the feed rate [1]. Pioneer researches [15, 21,38,23] have proved that considering variable processing speed into production scheduling is practical for resource-saving and time-efficient attempt. To address real85 world casting scenarios, the impact of variable processing speed for each operation is non-negligible. Research on scheduling problem with variable processing speed can be classified into two categories [22]: (1) continuous processing speed varying within a limited range;(2) discrete processing speed selecting from a predefined speed set. The discrete processing speed is suitable when considering practical applications [22] and is adapted in this paper.

Thus, the MOCPSP consists of three sub-problems: routing problem, sequencing problem and speed determination problem. 90 Former two are origin from classical FJSP [41]. Routing problem is to assign each operation to a machine out of a set of alternative machines. Sequencing problem is to sequence operation the assigned operations on all machines under the constraint. Speed determination problem is to select a processing speed of the assigned machine for each operation.

Therefore, the MOCPSP can be defined as follows:

There is a set of $n$ jobs $\boldsymbol{J}=\left\{J_{1}, J_{2}, \ldots, J_{n}\right\}$ and a set of $m$ machines $\boldsymbol{M}=\left\{M_{1}, M_{2}, \ldots, M_{m}\right\}$, and $M_{k}(k \in\{1,2, \ldots, m\})$ has $p_{k}$ 95 alternative speed ratios $\boldsymbol{\alpha}=\left\{\alpha_{1}^{k}, \alpha_{2}^{k}, \ldots, \alpha_{p_{k}}^{k}\right\}$ (the speed ratios increase gradually with the growth of index). $J_{i}(i \in\{1,2, \ldots, n\}$ ) contains the number of $s_{i}$ operations and each operation of $J_{i}$ follows the precedence relation. Operation $O_{i j}$ needs to be processed on one machine out of an alternative machine set $S_{i j}\left(S_{i j} \subseteq\{1,2, . ., M\}\right)$ containing at least one machine. And at least one operation can be processed on more than one machine.

Notations of the proposed MOCPSP are listed in Table 1

100 To simplify and clarify the proposed problem, some assumptions are predefined. 
Table 1 Notations of the proposed MOCPSP

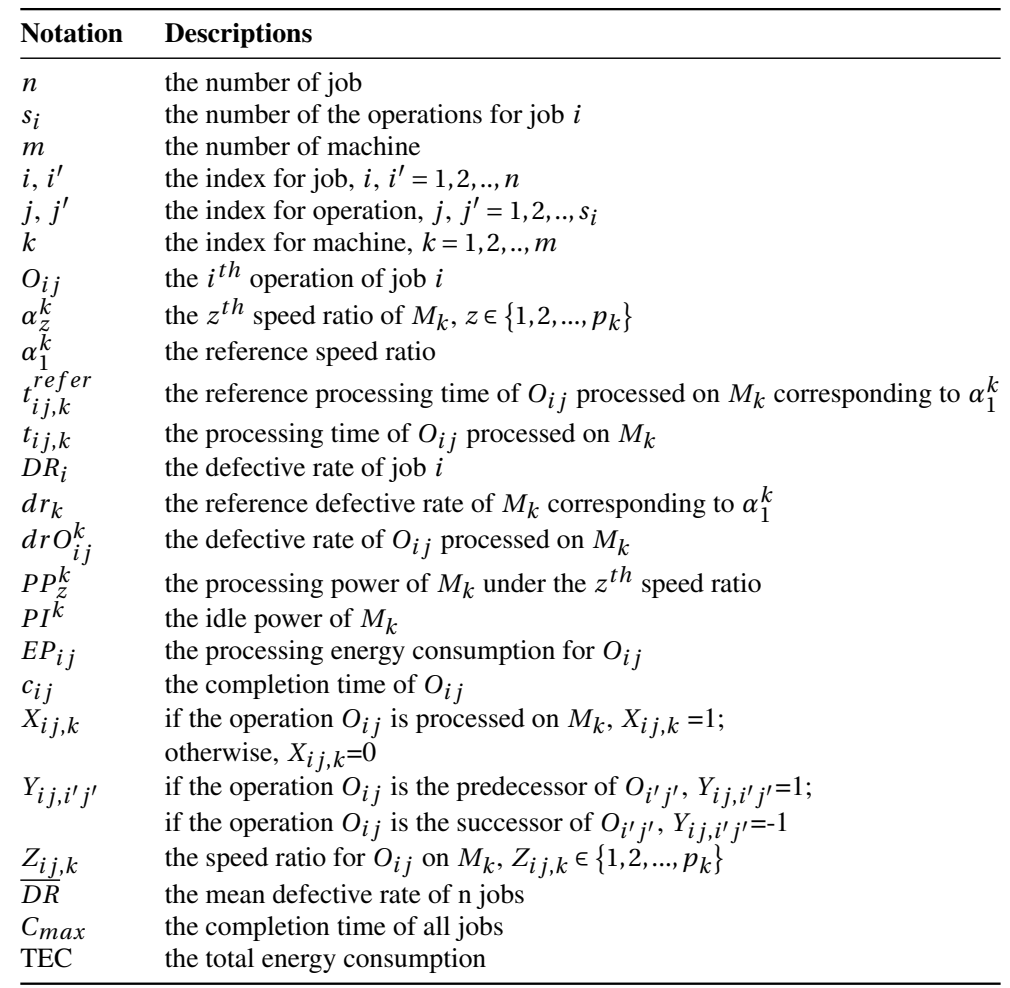

- All machines and raw materials are available at time 0;

- No setup operation for machine happens during production;

- Each operation is processed without interruption;

- The switch between processing mode and idle mode requires no time;

- Conversion time is negligible.

\subsection{Models of the three objectives}

The models of defective rate, makespan and total energy consumption are described as follows.

\subsubsection{The model of defective rate}

In general, the defective rate varies with the machine deterioration and production rate [27]. When the machine shifts to the 'out-of-control' state [16], some percentage of the items produced are defective and a faster production rate deteriorates the quality of the process [13,24]. Since casting production scheduling has minor production cycle, the degree of machine deterioration can be viewed as unchanged and the concept of production rate can be described as the processing speed of operation under the shop floor environment. In this regard, the defective rate is merely the function of processing speed in the addressed situation.

Defective rate of an operation is the possibility of this operation processed with defect. In the addressed casting shop floor scenario, a product is not defective only when all operations of it processed without defects. Therefore, the $D R_{i}$ can be calculated by Eq. (1).

$$
D R_{i}=1-\prod_{j=1}^{s_{i}}\left(1-\sum_{k=1}^{m} X_{i j, k} d r O_{i j}^{k}\right)
$$

Defective rate increases with the acceleration of processing speed. The idea in [20] is adapted and the defective rate is assumed to be a linear function of processing speed. If $O_{i j}$ is processed on $M_{k}$, then

$$
\frac{d r O_{i j}^{k}}{\alpha_{Z_{i j, k}}^{k}}=\frac{d r_{k}}{\alpha_{1}^{k}}
$$

120 With Eqs. (1) and 22, the mean defective rate $\overline{D R}$ of $n$ jobs is formulated as Eq. 3).

$$
\overline{D R}=\frac{\sum_{i=1}^{n}\left[1-\prod_{j=1}^{s_{i}}\left(1-\sum_{k=1}^{m} X_{i j, k} d r_{k} \frac{\alpha_{Z_{i j, k}}^{k}}{\alpha_{1}^{k}}\right)\right]}{n}
$$




\subsubsection{The model of makespan}

The objective of makespan $\left(C_{\max }\right)$ is modeled as the matter of routine [17], the formulation is shown as Eq.44).

$$
C_{\text {max }}=\max \left\{C_{i j}\right\}
$$

The processing time of $O_{i j}$ processed on $M_{k}$ is calculated by Eq.(5).

$$
t_{i j, k}=\frac{t_{i j, k}^{r e f e r}}{\alpha_{Z i j, k}^{k}}
$$

\subsubsection{The total energy consumption model}

For an operation, it is commonly assumed that higher processing speed will reduce the processing time while consuming more energy [5]. Machines are generally in two main modes once turning on: processing mode and idle mode. When the machine is processing, it is in active mode and the output power is referred as the processing power. When the machine is in idle time between two processing operations, low output power is needed to hold the machine which is referred as idle power. In this paper, processing power is increased with the acceleration of processing speed. The value of processing power is predefined corresponding to the discrete processing speed. If $O_{i j}$ is processed on $M_{k}$, the processing energy consumption is calculated by Eq. (6).

$$
E P_{i j}=P P_{Z_{i j, k}}^{k} \frac{t_{i j, k}^{r e f e r}}{\alpha_{Z_{i j, k}}^{k}}
$$

Idle power is much lower than processing power and is regarded to be constant for a machine. To simplify the problem, it is assumed that all the machines keep turning on before all jobs are finished.

Therefore, the total energy consumption(TEC) is formulated as Eq. (7).

$$
T E C=\sum_{k=1}^{m} \sum_{i=1}^{n} \sum_{j=1}^{s_{i}} P P_{Z_{i j, k}}^{k} X_{i j, k} \frac{t_{i j, k}^{r e f e r}}{\alpha_{Z_{i j, k}}^{k}}+\sum_{k=1}^{m}\left(C_{\text {max }}-\sum_{i=1}^{n} \sum_{j=1}^{s_{i}} X_{i j, k} \frac{t_{i j, k}^{r e f e r}}{\alpha_{Z_{i j, k}}^{k}}\right) P I^{k}
$$

\subsection{The model of MOCPSP}

In the MOCPSP, the defective rate, the makespan and the total energy consumption are optimized simultaneously, shown as Eqs. (8) 111).

s.t.

$$
\begin{gathered}
\sum_{k=1}^{m} X_{i j, k}=1, \forall i, j, k \subseteq S_{i j} \\
C_{i j}-C_{i(j-1)} \geq \frac{t_{i j, k}^{r e f e r}}{\alpha_{Z_{i j, k}}^{k}} X_{i j, k}, j=1,2, \ldots, s_{i} \\
\left(C_{i^{\prime} j^{\prime}}-C_{i j}-\frac{t_{i^{\prime} j^{\prime}, k}^{r e f e r}}{\alpha_{Z_{i^{\prime} j^{\prime}, k}}^{k}}\right) X_{i j, k} X_{i^{\prime} j^{\prime}, k}\left(Y_{i j, i^{\prime} j^{\prime}}+1\right)+\left(C_{i j}-C_{i^{\prime} j^{\prime}}-\frac{t_{i j, k}^{r e f e r}}{\alpha_{Z_{i j, k}}^{k}}\right) X_{i j, k} X_{i^{\prime} j^{\prime}, k}\left(1-Y_{i j, i^{\prime} j^{\prime}}\right) \geq 0, \forall i, j, i^{\prime}, j^{\prime}, k
\end{gathered}
$$

Eq. 9 ensures that one operation should only be processed once by one of the available machines. Eq. (10) guarantees the predefined precedence relation of operations. Eq. (11) represents that a machine can only process one operation at a time.

\section{The proposed N-NSGA-II for the MOCPSP}

NSGA-II [10] is one of the most popular multi-objective optimizers. The main features of NSGA-II are summed as follows:

(1) computational complexity is reduced by a faster non-dominated sorting algorithm.

(2) solution diversity is improved by utilizing crowding distant to evaluate the density of non-dominated solutions.

In the light of those advantages, NSGA-II has been successfully applied to tackle the continuous optimization problems [39. 2,34|. However, the MOCPSP is a discrete combinatorial optimization problem with extreme complexity, whose solution space is discontinuous and enormous. Utterly random local search mechanism of NSGA-II is time-costly and has poor convergence. 145 Therefore, in this paper the improvements of the proposed N-NSGA-II are mainly presented in the two aspects: enhanced local search by neighborhood structures and novel solution generating approach. The detail of proposed N-NSGA-II for MOCPSP is successively described in the following subsections. 
Table 2 The speed ratio, power and reference defective rate information of the $4 * 4$ instance

\begin{tabular}{|c|c|c|c|c|c|c|c|}
\hline \multirow{2}{*}{$\begin{array}{l}\text { Machine } \\
M_{1}\end{array}$} & \multicolumn{5}{|c|}{$\begin{array}{l}\text { Discrete speed ratio/processing power } \\
(\mathrm{kW})\end{array}$} & \multirow{2}{*}{$\begin{array}{l}\text { Idle power } \\
(\mathbf{k W})\end{array}$} & \multirow{2}{*}{$\begin{array}{l}\begin{array}{l}\text { Reference } \\
\text { defective rate }\end{array} \\
0.013\end{array}$} \\
\hline & $1 / 2.16$ & $1.1 / 2.68$ & $1.2 / 3.43$ & $1.3 / 4.11$ & $1.4 / 4.56$ & & \\
\hline$M_{2}$ & $1 / 1.68$ & $1.2 / 2.32$ & $1.4 / 3.09$ & $1.6 / 3.73$ & - & 0.19 & 0.002 \\
\hline$M_{3}$ & $1 / 2.54$ & $1.15 / 3.18$ & $1.3 / 4.21$ & $1.45 / 5.03$ & $1.6 / 6.61$ & 0.22 & 0.018 \\
\hline$M_{4}$ & $1 / 1.97$ & $1.3 / 2.65$ & $1.6 / 3.52$ & - & - & 0.23 & 0.030 \\
\hline
\end{tabular}

Table 3 The reference processing time information of the $4 * 4$ instance

\begin{tabular}{llllll}
\hline \multirow{2}{*}{ Job } & \multirow{2}{*}{ operation } & \multicolumn{5}{c}{ Reference processing time } \\
& & M1 & M2 & M3 & M4 \\
\hline \multirow{3}{*}{ Job1 } & $O_{11}$ & 5 & 4 & 9 & - \\
& $O_{12}$ & 11 & 20 & - & 14 \\
& $O_{13}$ & 8 & 8 & - & - \\
& $O_{21}$ & 14 & 22 & 16 & - \\
\multirow{5}{*}{ Job2 } & $O_{22}$ & - & 17 & 17 & 19 \\
& $O_{23}$ & 20 & - & 15 & - \\
& $O_{24}$ & 10 & - & - & 10 \\
\multirow{5}{*}{ Job3 } & $O_{31}$ & 8 & 12 & - & 10 \\
& $O_{32}$ & - & - & 6 & 6 \\
\multirow{3}{*}{ Job4 } & $O_{33}$ & 19 & 16 & - & 16 \\
& $O_{41}$ & - & 18 & - & 16 \\
& $O_{42}$ & 30 & 30 & 25 & - \\
\hline
\end{tabular}

$\begin{array}{llllllllllll}\boldsymbol{O}_{11} & \boldsymbol{O}_{12} & \boldsymbol{O}_{13} & \boldsymbol{O}_{21} & \boldsymbol{O}_{22} & \boldsymbol{O}_{23} & \boldsymbol{O}_{24} & \boldsymbol{O}_{31} & \boldsymbol{O}_{32} & \boldsymbol{O}_{33} & \boldsymbol{O}_{41} & \boldsymbol{O}_{42}\end{array}$

\begin{tabular}{|c|c|c|c|c|c|c|c|c|c|c|c|c|}
\hline $\mathbf{X}_{\mathrm{OS}}$ & 2 & 5 & 8 & 1 & 7 & 9 & 12 & 4 & 6 & 11 & 3 & 10 \\
\hline $\mathbf{X}_{\mathrm{MA}}$ & 2 & 4 & 1 & 3 & 2 & 3 & 1 & 4 & 4 & 1 & 2 & 3 \\
\hline $\mathbf{X}_{\mathrm{PSR}}$ & 0.3 & 0.7 & 0.1 & 0.1 & 0.4 & 0.6 & 0.7 & 0.4 & 0.6 & 0.5 & 0.4 & 0.8 \\
\hline
\end{tabular}

Fig. 1 An illustration of the coding method

\subsection{Coding and decoding methods}

In N-NSGA-II, three vectors are included corresponding to three decision variables: operation sequencing vector, machine assignment vector and speed ratio seed vector. They are represented by $X_{O S}, X_{M A}$ and $X_{S R S}$, the dimensions of which equal to the number of all operation to be processed.

The $X_{O S}$ is a permutation of the number of all operations. Note that the operations belong to the same job should order in ascending to avoid infeasible solution [7]. The gene in $X_{M A}$ is the machine number. The machine for the operation should be selected from the alternative set. The gene in $X_{S R S}$ is a random number range from 0 to 1 . The speed ratio seed of $O_{i j}$ is noted as $v_{i j}$. The speed ratio for $O_{i j}$ on $M_{k}$ is calculated by Eq. 12.

$$
Z_{i j, k}=v_{i j} \otimes p_{k}, v_{i j} \in(0,1]
$$

where $\otimes$ represents the ceil operator.

The "active" decoding is applied in this paper, the procedure of which is described concretely in [7].

To better demonstrate the coding and decoding methods, a case with 4 jobs and 4 machines $(4 * 4)$ is taken for instance. The speed ratio, power and reference defective rate information is shown in Table 2 and the reference processing time information is shown in Table 3 The three-vector coding is illustrated in Fig 1

Take $O_{22}$ as an example. The allocating sequence of $O_{22}$ is $7^{\text {th }}$. The machine for $O_{22}$ is $\mathrm{M}_{2}$, which has 4 discrete speed ratios. So $Z_{22,2}$ is calculated by Eq. 12 and value is 2, which means the $2^{\text {nd }}$ speed ratio of $M_{2}$ is selectd for $O_{22}$. And the defective rate, the processing time and processing power for $O_{22}$ are obtained by Eq.(2), Eq.(5) and Eq. (6) respectively.

The Gantt chart of the illustrated coding is shown in Fig 2 .

\section{$165 \quad 3.2$ Initialization}

For $X_{O S}$, the sequence of all genes is randomly initialized. For $X_{M A}$, Zhang et.al [44] developed an effective initialization method to assign operation to the suitable machine among which the two initial strategies named global selection (GS) and local selection (LS) are applied in this paper to initialize $X_{M A}$. The proportion of GS is referred as $r_{G S}$ and the proportion of LS is equal to $1-r_{G S}$. For $X_{S R S}$, the solution set is equally divided into three portions. The first portion is initialized with 1 for all genes, the second portion is initialized with 0 for all genes, the rest is randomly initialized for all genes. 


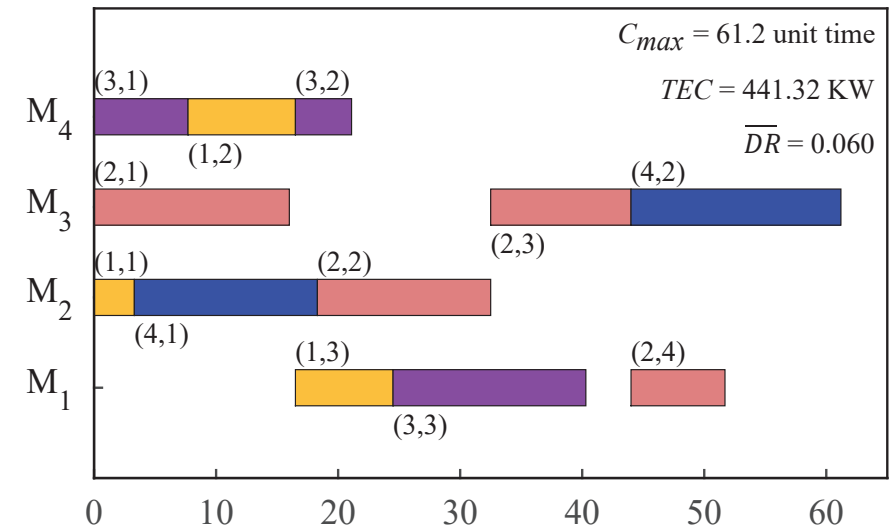

Fig. 2 The Gantt chart of the coding in Fig 1

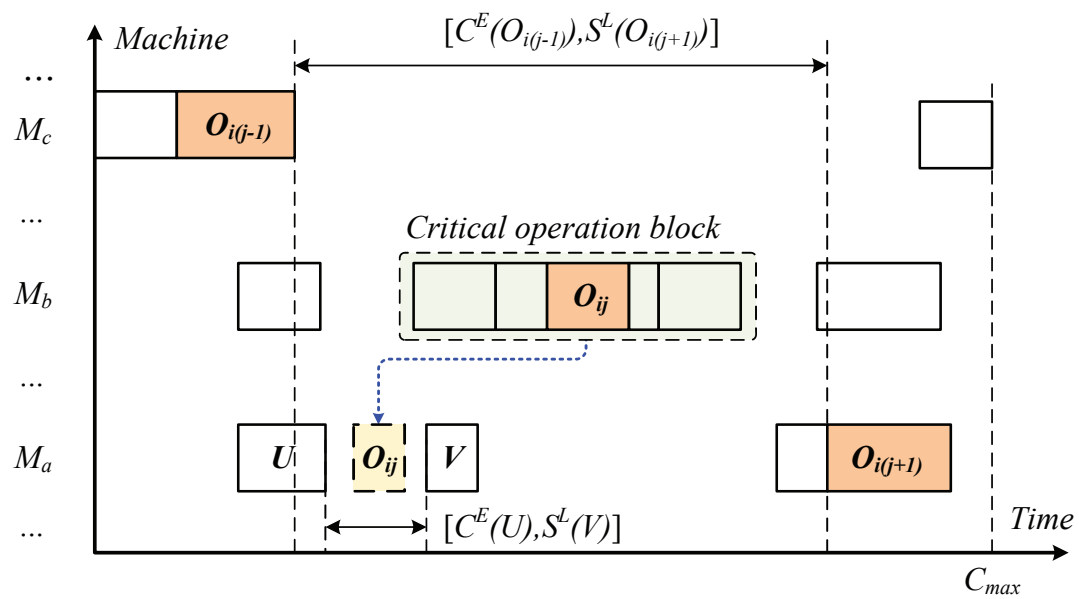

Fig. 3 Schematic of the NS1

\subsection{Enhanced local search by neighborhood structures}

Neighborhood structure embedded with the knowledge of MOCPSP serves as a powerful tool for local search. Let $N S_{k}(k \in$ $\left.\left\{1,2, \ldots, k_{\max }\right\}\right)$ to be one of the set of the $k_{\max }$ predefined neighborhood structures. $N S_{k}(s)$ is the set of neighbor solutions of $s$ in the $N S_{k}$.

In this paper, three neighborhood structures are designed corresponding to the three decision variables, denoted as $N S_{1}$, $N S_{2}$ and $N_{3}$. And the three decision variables can be divided into two categories: operation movements (decision variables $X$ and $Y$ ) and speed ratio seed (decision variable $Z$ ) mutation.

Operation movements are based on the concept of critical path, critical operation and critical operation block [46]. Operation movements including two situations: cross-machine movement and same-machine movement, corresponding to $N S_{1}$ and $N S_{2}$. Illustrations of $N S_{1}$ and $N S_{2}$ are shown in Fig 3 and Fig 4 respectively. $O_{i j}$ is a critical operation from a critical operation block containing at least two operations. $O_{i(j-1)}$ and $O_{i(j+1)}$ are the predecessor and successor operations of the same job adjacent to $O_{i j}$ respectively. $S^{L}$ represents the latest start time and $C^{E}$ represents the earliest completion time of an operation. Movement of $O_{i j}$ is limited by $O_{i(j-1)}$ and $O_{i(j+1)}$, whose range is $\left[C^{E}\left(O_{i(j-1)}\right), S^{L}\left(O_{i(j+1)}\right)\right]$.

$N S_{1}$ : cross-machine movement for operation is illustrated in Fig 3 . In MOCPSP, the number of machine should be more than one. Assume $U$ and $V$ are two consecutive operations on machine $M a$ ( $U$ is processed before $V$ ) and $O_{i j}$ is not processed on $M a$. If $M a$ is one of the alternative machines of $O_{i j}$ and Eq. (13) is satisfied, $O_{i j}$ is moved to $M a$. If no such movement exists, $O_{i j}$ is moved out to another random machine in the set of alternative machines.

$$
\begin{cases}{\left[0, S^{L}\left(O_{i(j+1)}\right)\right] \cap\left[C^{E}(U), S^{L}(V)\right]>\frac{t_{i j, M a}^{r e f e r}}{\alpha_{Z_{i j, M a}}^{M a}},} & j=1 \\ \left.\left[C^{E}\left(O_{i(j-1)}\right), C_{\text {max }}\right)\right] \cap\left[C^{E}(U), S^{L}(V)\right]>\frac{t_{i j, M a}^{\text {refer }}}{\alpha_{Z_{i j, M_{a}}^{M a}}^{M}}, & j=s_{i} \\ {\left[C^{E}\left(O_{i(j-1)}\right), S^{L}\left(O_{i(j+1)}\right)\right] \cap\left[C^{E}(U), S^{L}(V)\right]>\frac{t_{i j, M_{a}}}{\alpha_{Z_{i j, M_{a}}} M_{a}},} & \text { otherwise }\end{cases}
$$

$N S_{2}$ : same-machine movement for operation is illustrated in Fig 4 . The purpose of $N S_{2}$ is to move critical operation $O_{i j}$ out the critical operation block on the same machine to shorten the length of critical operation block. There are three situations: 1901 ) if $O_{i j}$ is the internal operation of the critical operation block, $O_{i j}$ would be moved before the first operation or after the last operation of the critical operation block within the range of $\left[C^{E}\left(O_{i(j-1)}\right), S^{L}\left(O_{i(j+1)}\right)\right]$, as shown in Fig 4 (a). 


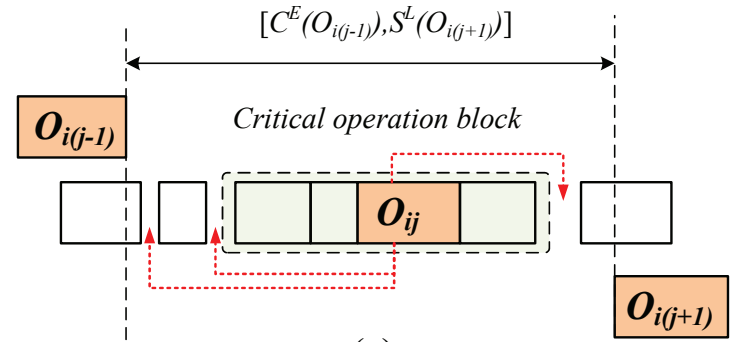

(a)

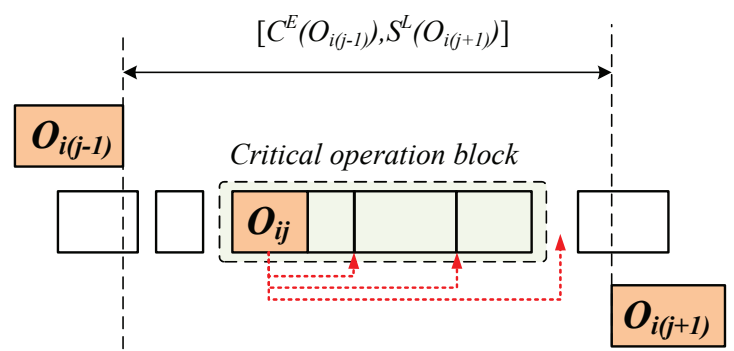

(b)

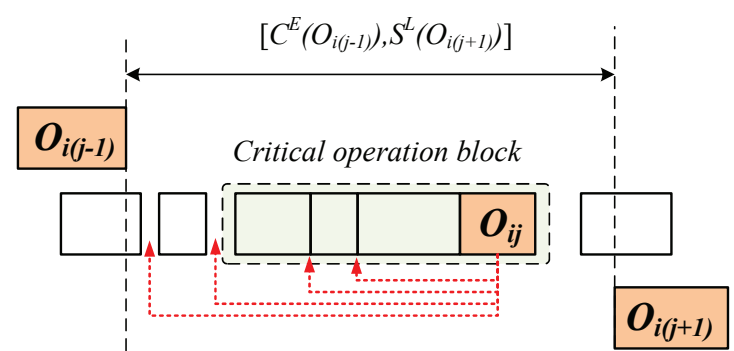

(c)

Fig. 4 Schematic of the NS2

2) if $O_{i j}$ is the start operation of the critical operation block, $O_{i j}$ would be moved backwards within the range of $\left[C^{E}\left(O_{i(j-1)}\right), S^{L}\left(O_{i(j+1)}\right)\right]$, as shown in Fig 4 (b).

3) if $O_{i j}$ is the end operation of the critical operation block, $O_{i j}$ would be moved forwards within the range of $\left[C^{E}\left(O_{i(j-1)}\right), S^{L}\left(O_{i(j+1)}\right)\right]$, as shown in Fig 4(c).

Speed ratio seed (decision variable $Z$ ) is adjusted by mutation operator. The detail is given as follows.

$N S_{3}$ : two parameters are defined: mutation probability $\left(p_{m}\right)$ and step size $(\sigma)$. $p_{m}$ determines the number of operation to mutate in operation string. Assume that one of the solutions is to mutate. Select another solution $S_{2}$ from the solution set as reference solution and the speed ratio seed of $O_{i j}$ in $\beta+1$ iteration is updated by Eq.(14).

$$
v_{i j}^{\beta+1}=\left(v_{i j}^{\beta, \text { refer }}-v_{i j}^{\beta}\right) \times \sigma \times \epsilon+v_{i j}^{\beta}
$$

Where $v_{i j}^{\beta, r e f e r}$ is the speed ratio seed of $O_{i j}$ of the reference solution, $\epsilon$ is a random number range from 0 to 1 .

\subsection{Elitism strategy}

To reserve more optimal solutions, the elitism strategy is adapted in the N-NSGA-II. The reservation takes place after local search. In the first iteration, the first Pareto front solutions are viewed as elites and reserved. In the following iterations, the first Pareto front solutions found in the current iteration are combined with the former elites, and solutions in the new first Pareto front are viewed as elites and reserved.

\subsection{Novel solution generating approach}

To improve the diversity of solution, a novel solution generating approach is designed in N-NSGA-II. The illustration of the proposed solution generating approach is shown in Fig 5 .

Step1-Inherit the genes from solution set. For a new solution, the gene of each operation is inherited from the gene of the same operation of a solution randomly selected from solution set. As shown in Fig $5, O_{11}, O_{12}, O_{21}, O_{22}$ of the new solution are inherited from $S_{2}, S_{3}, S_{3}, S_{2}$ respectively. Repeat the procedure until generating $N p o p_{S G}$ new solutions. 


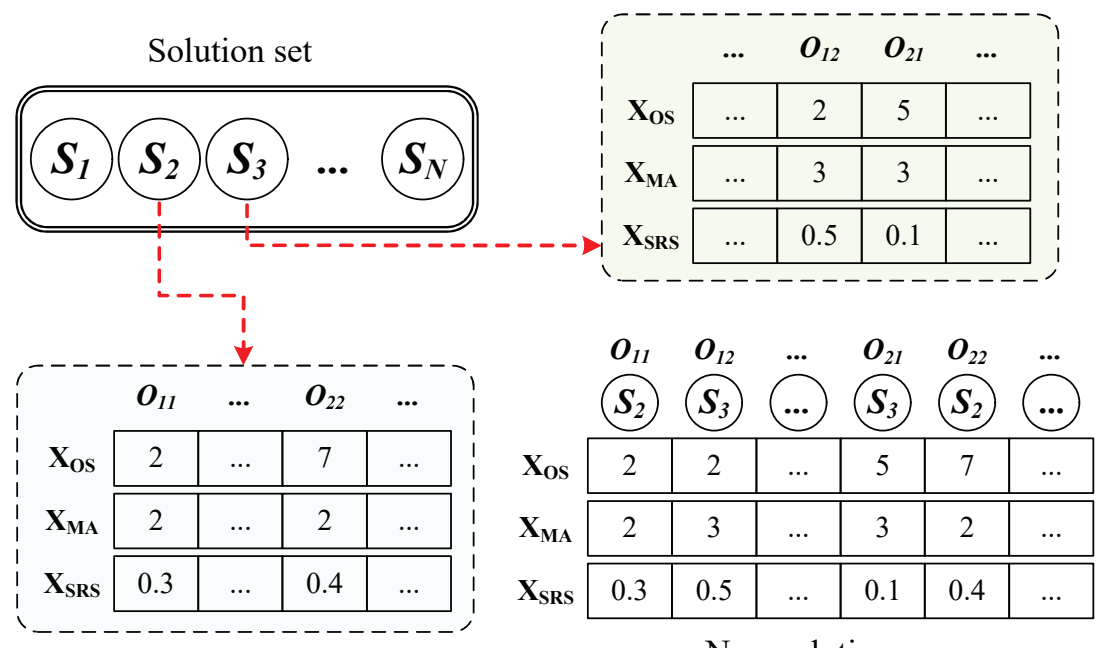

New solution

Fig. 5 Schematic of the novel solution generating approach

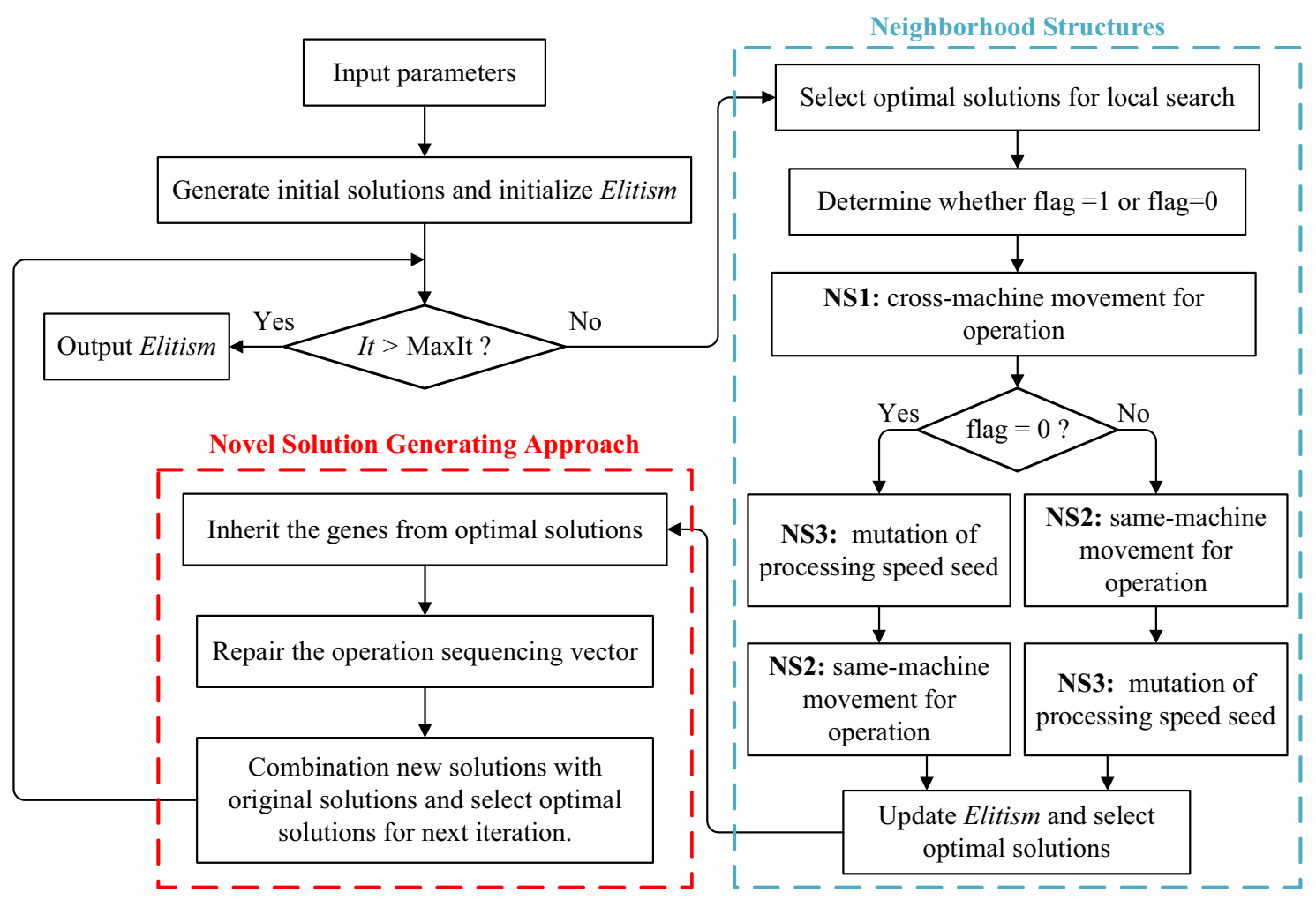

Fig. 6 Flowchart of the proposed N-NSGA-II

Step2-Repair the $X_{O S}$ vector. When there is the repeated number in $X_{O S}$ vector, as shown in Fig 5 the repair is needed to avoid infeasible solution. Repair follows the rules:(1) The operations with the same $X_{O S}$ gene are random sorted;(2) The operation with larger $X_{O S}$ gene comes after the smaller one. The procedure is described as follows:

(1) Sort $X_{O S}$ of all operations with by ascending.

(2) For the operations with the same $X_{O S}$ gene, switch their positions randomly.

(3) Number all operations successively with their positions, the first position is numbered 1.

(4) Update the $X_{O S}$ with the corresponding operation number.

Step3-Combination and selection. Combine the $N p p_{S G}$ new solutions with the initial solution set to form a new solution set and utilize the selection mechanism of NSGA-II to select $N p o p$ optimal solutions for next iteration.

\subsection{Stopping criterion}

The algorithm terminates after a certain number of iterations. This number is predefine and referred as MaxIt. 


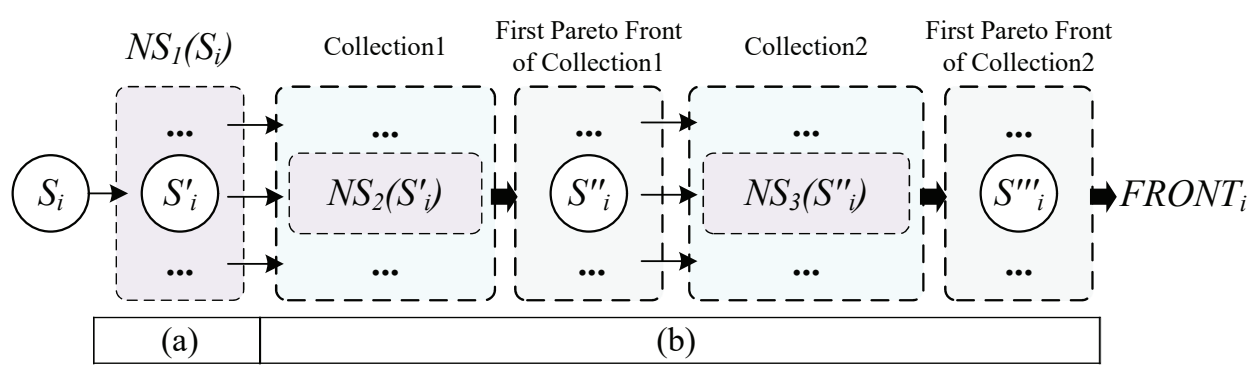

Fig. 7 Schematic of local search by the three neighborhood structures(flag=1)

\subsection{The procedure of N-NSGA-II}

The flowchart of the proposed N-NSGA-II is illustrated in Fig 5 The overall procedure is described as follows.

Input parameters: MaxIt, $r_{G S}, N p o p, N p o p_{L}, N p o p_{S G}, p_{m}, \sigma, \tau$

Step1. Initialization: Generate $N p o p$ initial solutions by initialization approach in 3.2 .

Step2. Stopping criterion: Determine whether the stopping criterion is met. If not, go to step3. If so, output the Elitism.

Step3. Local search by neighborhood structures: Select the $\operatorname{Npop}_{L}\left(N p o p_{L}<N p o p\right)$ optimal solutions by the selection mechanism of NSGA-II. Perform the local search with the designed neighborhood structures for the optimal solutions. For solution $S_{i}$, the local search procedures are:

Step3-1. Generate a random number $r$ ranging from 0 to 1 . If $r$ is greater than the search constant $\tau$, flag=1; otherwise, flag=0. The $F R O N T_{i}$ is used to temporally reserve first Pareto front solutions found for $S_{i}$.

Step3-2. Find all neighbor solutions of $S_{i}$ in $N S_{1}$ and output the solution set $N S_{1}\left(S_{i}\right)$ to Step3-3, as shown in Fig 7 a).

Step3-3. If flag equals to 1, the procedure of local search by $N S_{2}$ and $N S_{3}$ successively is illustrated in Fig 7b): (1)find all neighbor solutions of each solution from $N S_{1}\left(S_{i}\right)$ in $N S_{2}$, which make up Collection1, and reserve the first Pareto front solutions of Collection1, (2)find all neighbor solutions of each solution from Collection1 in $N S_{3}$, which make up the Collection2, and reserve the first Pareto front solutions of Collection2, (3)output all solutions in Collection2 to $F R O N T_{i}$. For the circumstance that flag equals to 0 , switch the sequence of $N S_{2}$ and $N S_{3}$ and follow the same procedure as the circumstance that flag equals to 1 .

Step3-4. If $S_{i}$ is not a member of $F R O N T_{i}$, select one solution from $F R O N T_{i}$ and name it as $S_{i}$, return to Step3-2. Otherwise, output $F R O N T_{i}$.

Step4. Elitism strategy: Combine all $F R O N T_{i}$ s as well as the initial solution of this iteration with Elitism and perform the selection of NSGA-II [10]. Update the Elitism with the first Pareto front solutions of the combination and output the Npop optimal solutions to step 5 .

Step5. Novel solution generating approach: Perform novel solution generating approach in 3.5 , output $N p o p$ solutions and return to step 2 .

\section{Computational experiments and discussions}

In this section, a real-world casting production scheduling case is investigated. Four performance metrics for measuring the Pareto front obtained by algorithms are employed. Then, the effectiveness of the neighborhood structures and the novel solution to evaluate the performance of N-NSGA-II on the MOCPSP. All algorithms are coded in Matlab R2018b and run on a computer with Intel Core i7(3.0GHz), 8 GB RAM under Windows 10 operation environment.

\subsection{Case description}

To consider MOCSPS in the real-world casting workshop, a case with 20 jobs and 15 machines is presented. In this case, 15 common operations are addressed and the machines for each operation are listed in Table 4. The process route of each job and the corresponding reference processing time information are shown in Table 5 The speed ratio, power and reference defective rate information of machines are listed in Table 6 .

\subsection{Performance metrics}

To give quantitative evaluations on the performance of multi-objective optimization algorithms, four metrics are employed including generational distance (GD), contribution rate (CR), inverse generational distance (IGD) and set coverage( $c$-metric). The definitions of the four metrics are listed as follows. 
Table 4 Operations and alternative machines of the real-world casting case

\begin{tabular}{|c|c|c|c|c|}
\hline \multicolumn{5}{|l|}{ Operation } \\
\hline 01 & O2 & O3 & O4 & O5 \\
\hline Modling & Melting & Clearing & Thermal treatment & Rough turning \\
\hline$\left[M_{1}, M_{2}\right]$ & $M_{3}$ & {$\left[M_{4}, M_{5}\right]$} & $M_{6}$ & {$\left[M_{7}, M_{8}, M_{9}\right]$} \\
\hline 06 & 07 & 08 & 09 & 010 \\
\hline Semi-extractive turning & Extractive turning & Fine turning & Rough milling & Semi-extractive milling \\
\hline$\left[M_{8}, M_{9}\right]$ & {$\left[M_{8}, M_{9}\right]$} & {$\left[M_{9}\right]$} & {$\left[M_{8}, M_{9}, M_{10}, M_{11}, M_{12}\right]$} & {$\left[M_{10}, M_{11}\right]$} \\
\hline 011 & 012 & 013 & 014 & 015 \\
\hline Extractive milling & Weld repairing & Extractive grinding & Fine grinding & Flaw detection \\
\hline$M_{11}$ & $M_{13}$ & {$\left[M_{8}, M_{9}, M_{12}, M_{14}\right]$} & {$\left[M_{12}, M_{14}\right]$} & $M_{15}$ \\
\hline
\end{tabular}

\section{(1) Convergence metrics: GD [47]}

$$
G D=\frac{\sqrt{\sum_{i=1}^{|\Omega|} D_{i}^{2}\left(\Omega, \Omega^{*}\right)}}{|\Omega|}
$$

where $\Omega^{*}$ is the true first Pareto front(TFPF) and $\Omega$ is the first Pareto front found by an algorithm. $D_{i}\left(\Omega, \Omega^{*}\right)$ is the shortest Euclidean distance between the solution $i$ in $\Omega$ and the solutions in $\Omega^{*}$.

(2) Quality metric: CR [29]

$$
C R=\frac{\left|\left\{x \mid x \in \Omega \& x \in \Omega^{*}\right\}\right|}{\left|\Omega^{*}\right|} \times 100 \%
$$

(3) Comprehensive metric: IGD [48]

$$
I G D=\frac{\sum_{i=1}^{\left|\Omega^{*}\right|} D_{i}\left(\Omega^{*}, \Omega\right)}{\left|\Omega^{*}\right|}
$$

where $D_{i}\left(\Omega^{*}, \Omega\right)$ is the shortest Euclidean distance between the solution $i$ in $\Omega^{*}$ and the solutions in $\Omega$.

(4) Set coverage metric: $c$-metric [48]

$$
C(P, Q)=\frac{|\{q \in Q: \exists p \in P, p>q\}|}{Q}
$$

where $P$ and $Q$ are the first Pareto fronts obtained by two algorithms, $p>q$ represents the $q$ is dominated by $p$.

The smaller value of GD and IGD while the bigger value of CR represents good performance. And the bigger value of

\begin{tabular}{|c|c|c|c|c|c|c|c|c|}
\hline \multirow{2}{*}{$\overline{\bar{J}} \overline{\text { Job1 }}$} & \multicolumn{8}{|c|}{ Process route of each job and the reference processing time on the alternative machines } \\
\hline & $\mathbf{0 1}$ & $\mathbf{O 2}$ & $\mathbf{O 3}$ & $\mathbf{O 4}$ & $\mathbf{O 5}$ & 09 & $\mathbf{O 1 3}$ & O6 \\
\hline & {$[46,35]$} & 6 & {$[16,17]$} & 33 & {$[29,34,11]$} & {$[34,16,25,13,26]$} & {$[26,16,27,13]$} & {$[30,15]$} \\
\hline & 07 & 012 & 014 & 010 & 011 & 08 & 015 & \\
\hline & {$[37,19]$} & 14 & {$[31,11]$} & {$[26,11]$} & 11 & 20 & 17 & \\
\hline \multirow[t]{4}{*}{ Job2 } & 01 & $\mathbf{O 2}$ & $\mathbf{O 3}$ & O4 & 013 & $\mathbf{O 5}$ & O6 & $\mathbf{O 7}$ \\
\hline & {$[55,27]$} & 8 & {$[26,18]$} & 41 & {$[27,13,27,13]$} & {$[33,37,15]$} & {$[37,15]$} & {$[39,15]$} \\
\hline & 014 & O9 & $\mathbf{O 1 0}$ & 012 & O8 & 011 & 015 & \\
\hline & {$[33,11]$} & {$[28,12,33,11,26]$} & {$[28,17]$} & 13 & 18 & 16 & 8 & \\
\hline \multirow[t]{4}{*}{ Job3 } & 01 & $\mathbf{O 2}$ & $\mathbf{O 3}$ & O4 & 013 & O9 & 012 & 010 \\
\hline & {$[58,34]$} & 5 & {$[23,15]$} & 36 & {$[34,16,29,12]$} & {$[26,18,23,12,28]$} & 13 & {$[24,18]$} \\
\hline & 014 & O5 & O6 & O7 & O8 & 011 & 015 & \\
\hline & {$[30,13]$} & {$[30,43,11]$} & {$[44,15]$} & {$[43,15]$} & 16 & 15 & 7 & \\
\hline \multirow[t]{4}{*}{ Job4 } & 01 & $\mathbf{O 2}$ & $\mathbf{O 3}$ & 04 & O9 & O5 & 010 & 011 \\
\hline & {$[46,26]$} & 4 & {$[18,12]$} & 30 & {$[30,16,27,18,35]$} & {$[32,34,14]$} & {$[27,15]$} & 15 \\
\hline & O6 & 013 & O7 & 014 & O8 & 012 & 015 & \\
\hline & {$[31,11]$} & {$[38,12,31,12]$} & {$[43,13]$} & {$[30,11]$} & 12 & 13 & 7 & \\
\hline \multirow[t]{4}{*}{ Job5 } & 01 & $\mathbf{O 2}$ & $\mathbf{O 3}$ & 04 & 05 & 013 & O9 & O6 \\
\hline & {$[37,27]$} & 8 & {$[19,16]$} & 30 & {$[27,27,17]$} & {$[43,11,26,11]$} & {$[30,18,29,17,30]$} & {$[34,18]$} \\
\hline & 012 & $\mathbf{0 7}$ & O8 & $\mathbf{O 1 0}$ & 011 & 014 & 015 & \\
\hline & 15 & {$[43,17]$} & 20 & {$[23,14]$} & 12 & {$[33,15]$} & 8 & \\
\hline \multirow[t]{3}{*}{ Job6 } & 01 & $\mathbf{O 2}$ & $\mathbf{O 3}$ & 04 & O9 & O5 & 010 & O6 \\
\hline & {$[39,28]$} & 7 & {$[27,13]$} & 45 & {$[26,16,25,14,34]$} & {$[30,30,11]$} & {$[38,11]$} & {$[41,15]$} \\
\hline & 011 & 012 & O7 & 013 & 014 & 018 & 015 & \\
\hline
\end{tabular}
$C(P, Q)$ than $C(Q, P)$ represents the $P$ is preferable. Since the TFPF of the case is unknown, the first Pareto front of the solutions found by all employed algorithms is viewed as the TFPF in this paper.

Table 5: Process route and the corresponding reference processing time information 


\begin{tabular}{|c|c|c|c|c|c|c|c|c|}
\hline & \multicolumn{8}{|c|}{ Process route of each job and the reference processing time on the alternative machines } \\
\hline & 15 & [15] & {$[38,13]$} & {$[32,20,34,12]$} & {$[31,15]$} & 13 & 11 & \\
\hline \multirow[t]{4}{*}{ Job7 } & 01 & $\mathbf{O 2}$ & $\mathbf{O 3}$ & 04 & O9 & $\mathbf{O 1 3}$ & $\mathbf{O 1 0}$ & $\mathbf{O 5}$ \\
\hline & {$[55,41]$} & 4 & {$[18,11]$} & 31 & {$[38,17,35,16,35]$} & {$[38,17,32,15]$} & {$[22,15]$} & {$[28,35,15]$} \\
\hline & 06 & 014 & 011 & 07 & 012 & 08 & 015 & \\
\hline & {$[42,17]$} & {$[35,12]$} & 18 & {$[33,18]$} & 15 & 16 & 12 & \\
\hline \multirow{4}{*}{ Job8 } & 01 & $\mathbf{O 2}$ & $\mathbf{O 3}$ & O4 & $\mathbf{O 5}$ & O9 & O6 & 013 \\
\hline & {$[45,17]$} & 7 & {$[18,11]$} & 37 & {$[27,39,12]$} & {$[32,19,28,18,34]$} & {$[37,19]$} & {$[42,11,32,14]$} \\
\hline & 010 & O7 & 012 & 014 & 011 & O8 & 015 & \\
\hline & {$[29,14]$} & {$[31,19]$} & 14 & {$[28,15]$} & 17 & 19 & 10 & \\
\hline \multirow[t]{4}{*}{ Job9 } & 01 & $\mathbf{O 2}$ & $\mathbf{O 3}$ & 04 & $\mathbf{O 5}$ & 013 & O9 & O6 \\
\hline & {$[63,43]$} & 4 & {$[23,19]$} & 32 & {$[33,40,14]$} & {$[36,20,26,13]$} & {$[43,16,37,15,33]$} & {$[27,19]$} \\
\hline & 014 & 010 & 07 & 012 & 011 & O8 & 015 & \\
\hline & {$[33,11]$} & {$[31,16]$} & {$[42,13]$} & 13 & 16 & 20 & 13 & \\
\hline \multirow[t]{4}{*}{ Job10 } & 01 & $\mathbf{O 2}$ & $\mathbf{O 3}$ & O4 & 013 & $\mathbf{O 5}$ & O6 & O9 \\
\hline & {$[43,29]$} & 4 & {$[22,16]$} & 32 & {$[42,13,30,14]$} & {$[28,42,13]$} & {$[43,18]$} & {$[26,18,29,15,26]$} \\
\hline & 07 & 012 & 010 & 014 & 08 & 011 & 015 & \\
\hline & {$[35,16]$} & 13 & {$[21,16]$} & {$[27,13]$} & 14 & 12 & 18 & \\
\hline \multirow{4}{*}{ Job11 } & 01 & $\mathbf{O 2}$ & $\mathbf{O 3}$ & 04 & 013 & 05 & 012 & O6 \\
\hline & {$[44,45]$} & 4 & {$[30,16]$} & 44 & {$[36,17,29,12]$} & {$[26,38,12]$} & 11 & {$[44,20]$} \\
\hline & 07 & 014 & O8 & O9 & 010 & 011 & 015 & \\
\hline & {$[27,19]$} & {$[30,11]$} & 16 & {$[40,12,22,18,30]$} & {$[24,16]$} & 15 & 16 & \\
\hline \multirow[t]{4}{*}{ Job12 } & 01 & $\mathbf{O 2}$ & $\mathbf{O 3}$ & 04 & O9 & 05 & O6 & 012 \\
\hline & {$[63,26]$} & 7 & {$[26,20]$} & 43 & {$[39,11,36,14,35]$} & {$[30,37,15]$} & {$[44,16]$} & 15 \\
\hline & 013 & 010 & O7 & 011 & 08 & 014 & 015 & \\
\hline & {$[41,13,31,11]$} & {$[36,15]$} & {$[37,18]$} & 16 & 17 & {$[30,15]$} & 7 & \\
\hline \multirow[t]{4}{*}{ Job13 } & 01 & $\mathrm{O2}$ & $\mathbf{O 3}$ & O4 & 012 & O9 & $\mathbf{O 5}$ & 013 \\
\hline & {$[56,45]$} & 7 & {$[19,16]$} & 37 & 11 & {$[36,11,27,13,32]$} & {$[28,44,13]$} & {$[27,12,32,13]$} \\
\hline & 06 & 014 & 010 & 07 & 011 & 08 & 015 & \\
\hline & {$[41,14]$} & {$[30,11]$} & {$[35,13]$} & {$[31,18]$} & 12 & 11 & 9 & \\
\hline \multirow[t]{4}{*}{ Job14 } & 01 & $\mathbf{O 2}$ & $\mathbf{O 3}$ & 04 & O9 & 013 & $\mathbf{O 5}$ & 014 \\
\hline & {$[49,12]$} & 6 & {$[17,12]$} & 44 & {$[29,15,29,18,30]$} & {$[26,18,33,11]$} & {$[31,31,13]$} & {$[33,15]$} \\
\hline & 010 & O6 & 011 & 07 & O8 & 012 & 015 & \\
\hline & {$[12,16]$} & {$[28,13]$} & 11 & {$[44,13]$} & 13 & 15 & 16 & \\
\hline \multirow[t]{4}{*}{ Job15 } & 01 & $\mathbf{O 2}$ & $\mathbf{O 3}$ & 04 & O9 & $\mathbf{0 5}$ & 013 & 014 \\
\hline & {$[65,45]$} & 6 & {$[20,20]$} & 39 & {$[45,16,21,17,28]$} & {$[31,35,20]$} & {$[30,19,35,12]$} & {$[29,13]$} \\
\hline & 010 & 011 & O6 & 07 & 012 & 018 & 015 & \\
\hline & {$[21,16]$} & 18 & {$[34,17]$} & {$[36,11]$} & 14 & 19 & 18 & \\
\hline \multirow[t]{4}{*}{ Job16 } & 01 & $\mathbf{O 2}$ & $\mathbf{O 3}$ & 04 & O9 & 012 & 010 & $\mathbf{O 5}$ \\
\hline & {$[54,29]$} & 8 & {$[22,19]$} & 29 & {$[39,15,27,13,31]$} & 13 & {$[34,13]$} & {$[27,30,19]$} \\
\hline & 013 & O6 & O7 & 014 & O8 & 011 & 015 & \\
\hline & {$[37,14,32,12]$} & {$[40,18]$} & {$[35,17]$} & {$[31,11]$} & 19 & 17 & 19 & \\
\hline \multirow[t]{4}{*}{ Job17 } & 01 & $\mathbf{O 2}$ & $\mathbf{O 3}$ & 04 & 013 & $\mathbf{O 5}$ & O6 & O9 \\
\hline & {$[44,45]$} & 8 & {$[18,11]$} & 31 & {$[26,17,33,15]$} & {$[29,33,18]$} & {$[30,18]$} & {$[45,20,34,15,34]$} \\
\hline & 014 & O7 & O8 & 010 & 011 & 012 & 015 & \\
\hline & {$[34,12]$} & {$[35,13]$} & 20 & {$[25,12]$} & 14 & 14 & 19 & \\
\hline \multirow[t]{4}{*}{ Job18 } & 01 & $\mathbf{O 2}$ & $\mathbf{O 3}$ & 04 & $\mathbf{O 5}$ & O9 & 013 & O6 \\
\hline & {$[43,35]$} & 6 & {$[16,18]$} & 35 & {$[33,37,11]$} & {$[32,19,28,13,29]$} & {$[36,12,29,14]$} & {$[29,19]$} \\
\hline & 014 & 010 & O7 & O8 & 011 & 012 & 015 & \\
\hline & {$[32,11]$} & {$[31,13]$} & {$[43,19]$} & 12 & 11 & 11 & 12 & \\
\hline \multirow[t]{4}{*}{ Job19 } & 01 & $\mathbf{O 2}$ & $\mathbf{O 3}$ & 04 & 013 & $\mathbf{O 5}$ & O6 & O9 \\
\hline & {$[40,32]$} & 5 & {$[27,20]$} & 40 & {$[33,15,33,11]$} & {$[33,31,19]$} & {$[29,19]$} & {$[35,11,32,14,27]$} \\
\hline & 010 & O7 & 014 & 011 & 08 & 012 & 015 & \\
\hline & {$[31,17]$} & {$[31,14]$} & {$[32,13]$} & 13 & 19 & 13 & 9 & \\
\hline \multirow[t]{4}{*}{ Job20 } & 01 & $\mathbf{O} 2$ & $\mathbf{O 3}$ & O4 & O9 & 010 & 011 & 012 \\
\hline & {$[56,39]$} & 8 & {$[23,13]$} & 35 & {$[36,13,33,11,32]$} & {$[27,13]$} & 18 & 14 \\
\hline & 05 & O6 & O7 & O8 & 013 & 014 & 015 & \\
\hline & {$[31,29,18]$} & {$[31,15]$} & {$[38,11]$} & 18 & {$[28,13,26,13]$} & {$[33,13]$} & 11 & \\
\hline
\end{tabular}


Table 6 The speed ratio, power and reference defective rate information of machines

Table 7 Parameter settings of the N-NSGA-II and its variants

\begin{tabular}{lllll}
\hline \multirow{2}{*}{ Parameters } & $\boldsymbol{A}$ & $\boldsymbol{B}$ & $\boldsymbol{C}$ & $\boldsymbol{D}$ \\
& N-NSGA-II & NSGA-II+Novel SGA & NSGA-II+NS & NSGA-II \\
\hline Population size $($ Npop $)$ & 80 & 80 & 80 & 80 \\
Maximum iterations(MaxIt) & 50 & 500 & 50 & 500 \\
Proportion of GS in initialization $\left(r_{G S}\right)$ & 0.4 & 0.4 & 0.4 & 0.4 \\
Crossover+mutation operators $[43]$ & None & None & $\checkmark$ & $\checkmark$ \\
Crossover probability & - & - & 0.7 & 0.7 \\
Mutation probability & - & - & 0.4 & 0.4 \\
Neighborhood structures & $\checkmark$ & None & $\checkmark$ & None \\
Population size of NS $\left(\right.$ Npop $\left.p_{L}\right)$ & 20 & - & 20 & - \\
Mutation probability $\left(p_{m}\right)$ & 0.1 & - & 0.1 & - \\
Step size $(\sigma)$ & 0.5 & - & 0.5 & - \\
search constant $(\tau)$ & 0.6 & - & 0.6 & - \\
Novel SGA & $\checkmark$ & $\checkmark$ & None & None \\
Population size of new solution by $\left(\right.$ N $\left._{\text {pop }} p_{S G}\right)$ & 40 & 40 & - & - \\
\hline
\end{tabular}

4.3 Effectiveness of the neighborhood structures and the novel solution generating approach

To verify the effectiveness of the proposed neighborhood structures(NS) and the novel solution generating approach(novel 275 SGA), the N-NSGA-II is compared with its variants. The parameter settings of the algorithms are list in Table 7 . All the algorithms run 20 times independently and the elapsed time is approximately 500s(CPU time) per time with the parameter setting. The computational results on metrics of GD, CR and IGD are shown in Table 8 and on $c$-metric are shown in Table.9. $A, B, C$ and $D$ denote the N-NSGA-II, NSGA-II+Novel SGA, NSGA-II+NS and NSGA-II respectively in Table 8 and Table 9

\subsubsection{Discussion on the effectiveness of the neighborhood structures}

To discuss the effectiveness of the neighborhood structures, $A$ and $B$ can be used as a group of comparison while $C$ and $D$ can be used as the other. It can be seen from Table 8 and Table 9 that $A$ is superior to $B$ while $C$ is superior to $D$ on all the metrics. As shown in Table 8 , both $A$ and $C$ have better value than their comparisons on the metrics of GD, CR and IGD. Moreover, as shown in Table 9, $\boldsymbol{c}(\boldsymbol{A}, \boldsymbol{B})>\boldsymbol{c}(\boldsymbol{B}, \boldsymbol{A})$ and $\boldsymbol{c}(\boldsymbol{C}, \boldsymbol{D})>\boldsymbol{c}(\boldsymbol{D}, \boldsymbol{C})$. The notable differences indicate that the enhanced local search by proposed neighborhood structures is effective for improving the performance of N-NSGA-II.

\subsubsection{Discussion on the effectiveness of the novel solution generating approach}

To discuss the effectiveness of the novel solution generating approach, $A$ and $C$ can be used as a group of comparison while $B$ and $D$ can be used as the other. It can be seen from Table 8 and Table 9 that $A$ is superior to $D$ while $B$ is superior to $D$ on all the metrics. A similar analysis as 4.3.1 can be made and it is shown that the novel solution generating approach has positive impact on the performance of N-NSGA-II.

\subsection{Comparisons of N-NSGA-II with other algorithms}

To further demonstrate the overall performance of the N-NSGA-II, the SPEA-II [48] and NSGA-III [9] are employed to compare with the N-NSGA-II. The parameter settings of SPEA-II and NSGA-III are listed in Table.10 while the parameter setting of N-NSGA-II is the same as listed in Table 7 The computational process is the same as that in 4.3 
Table 8 Computational results of N-NSGA-II and its variants on GD, CR and IGD

\begin{tabular}{llll}
\hline & GD & CR & IGD \\
\hline $\boldsymbol{A}$ & $8.65 \mathrm{e}-3$ & $52.1 \%$ & $1.09 \mathrm{e}-1$ \\
$\boldsymbol{B}$ & $1.23 \mathrm{e}-1$ & $12.3 \%$ & $1.48 \mathrm{e}-1$ \\
$\boldsymbol{C}$ & $2.73 \mathrm{e}-2$ & $30.8 \%$ & $1.21 \mathrm{e}-1$ \\
$\boldsymbol{D}$ & $1.86 \mathrm{e}-1$ & $4.8 \%$ & $1.90 \mathrm{e}-1$ \\
\hline
\end{tabular}

Table 9 Computational results of N-NSGA-II and its variants on $c$-metric

\begin{tabular}{llllllll}
\hline$c(A, B)$ & $c(B, A)$ & $c(C, D)$ & $c(D, C)$ & $c(A, C)$ & $c(C, A)$ & $c(B, D)$ & $c(D, B)$ \\
\hline $7.50 \mathrm{e}-1$ & 0 & $8.36 \mathrm{e}-1$ & 0 & $4.36 \mathrm{e}-1$ & $5.00 \mathrm{e}-2$ & $4.13 \mathrm{e}-1$ & $1.50 \mathrm{e}-1$ \\
\hline
\end{tabular}

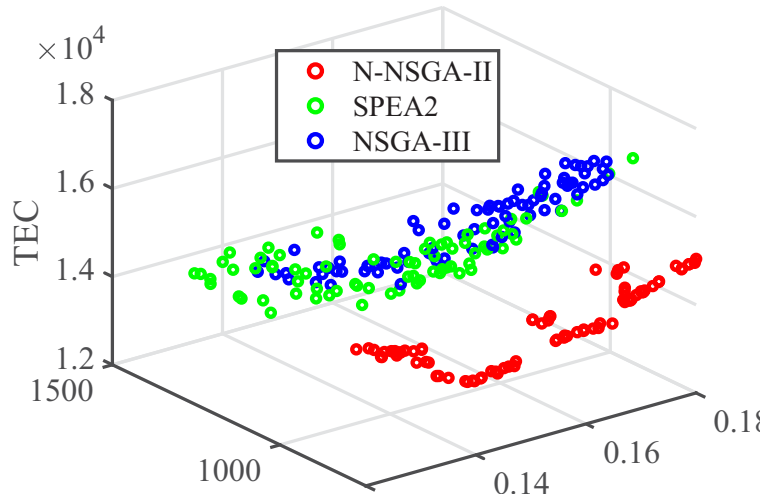

Makespan

DR (a)

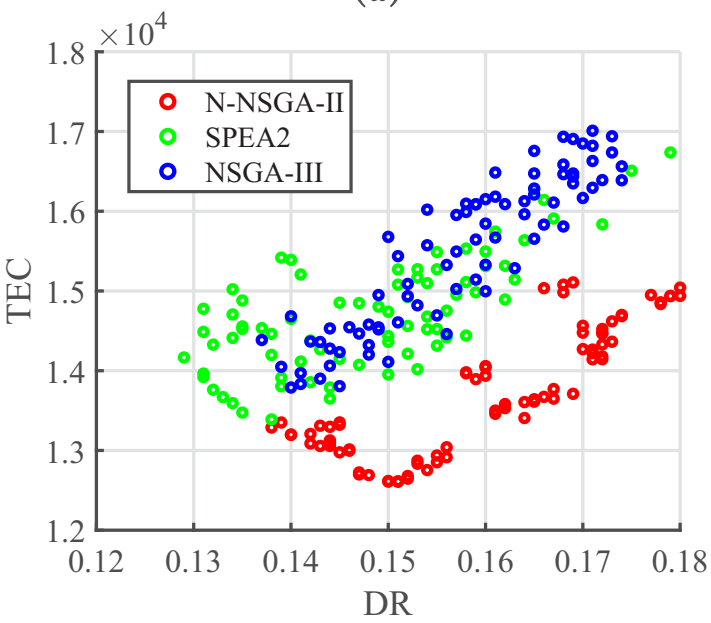

(c)

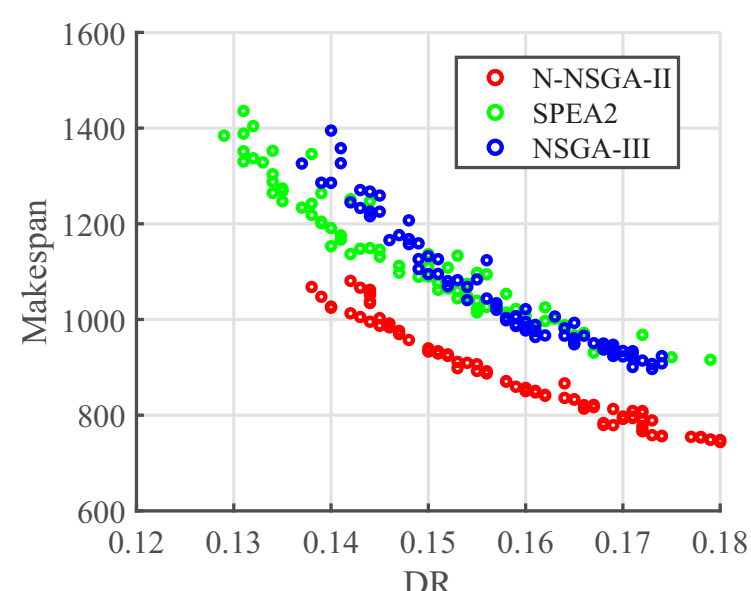

(b)

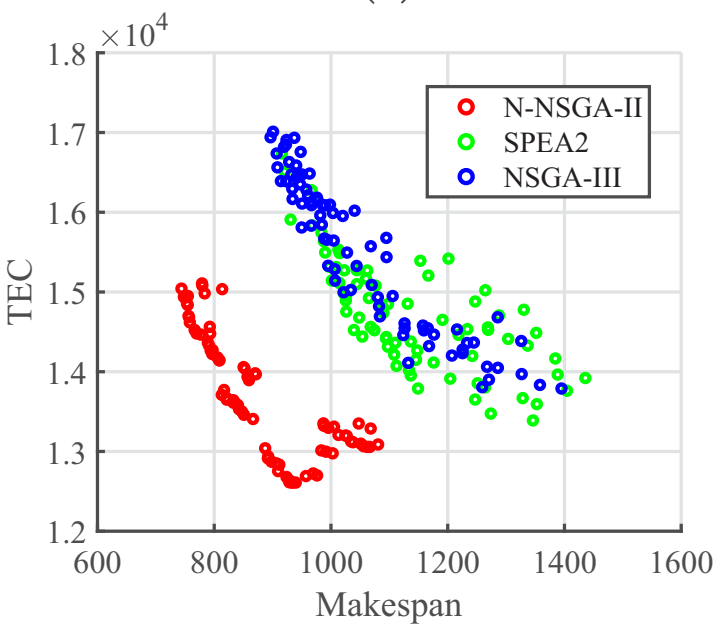

(d)

Fig. 8 The Pareto fronts obtained by N-NSGA-II, SPEA-II and NSGA-III under different perspectives

Table 10 Parameter settings of SPEA-II and NSGA-III

\begin{tabular}{|c|c|c|}
\hline & SPEA-II & NSGA-III \\
\hline Population size & 80 & 80 \\
\hline Maximum iterations & 500 & 500 \\
\hline Archive size 48 & 80 & - \\
\hline Number of division 9 & - & 5 \\
\hline \multicolumn{3}{|c|}{ Crossover+mutation operators 43} \\
\hline Crossover rate & 0.7 & 0.7 \\
\hline Mutation rate & 0.4 & 0.4 \\
\hline
\end{tabular}

The Pareto fronts obtained by N-NSGA-II, SPEA-II and NSGA-III under different perspectives are shown in Fig 8 It is cear that the N-NSGA-II approaches closer to the TFPF than the other two algorithms, which proves the superiority of the NNSGA-II. Fig. 8(a) shows a 3D view with three objectives represented by defective rate, makespan and total energy consumption respectively. It can be observed from Fig. 8 (b) that the defective rate is conflicted to the makespan. Moreover, defective rate of 
Table 11 Computational results of of N-NSGA-II, SPEA-II and NSGA-III on GD, CR and IGD

\begin{tabular}{llll}
\hline & GD & CR & IGD \\
\hline N-NSGA-II & $\mathbf{0}$ & $\mathbf{8 2 . 5 \%}$ & $\mathbf{1 . 1 1 e - 1}$ \\
SPEA-II & $2.10 \mathrm{e}-1$ & $17.5 \%$ & $2.83 \mathrm{e}-1$ \\
NSGA-III & $3.09 \mathrm{e}-1$ & 0 & $3.25 \mathrm{e}-1$ \\
\hline
\end{tabular}

Table 12 Computational results of N-NSGA-II, SPEA-II and NSGA-III on $c$-metric

\begin{tabular}{llll}
\hline$c(A, E)$ & $c(E, A)$ & $c(A, F)$ & $c(F, A)$ \\
\hline $7.76 \mathrm{e}-1$ & 0 & $9.86 \mathrm{e}-1$ & 0 \\
\hline
\end{tabular}

Table 13 Computational results of N-NSGA-II, SPEA-II and NSGA-III on $c$-metric

\begin{tabular}{|c|c|c|c|c|c|}
\hline No. & Defective rate & Makespan & $\begin{array}{l}\text { Total energy consumption } \\
\text { (kW) }\end{array}$ & Score & Obtained by \\
\hline 1 & 0.147 & 969.5 & $1.272 \mathrm{e}+4$ & 0.7531 & \\
\hline 2 & 0.148 & 957.1 & $1.267 \mathrm{e}+4$ & 0.7529 & \\
\hline 3 & 0.150 & 933.2 & $1.262 \mathrm{e}+4$ & 0.7526 & \\
\hline 4 & 0.147 & 976.2 & $1.270 \mathrm{e}+4$ & 0.7519 & \\
\hline 5 & 0.140 & 1024.6 & $1.320 \mathrm{e}+4$ & 0.7517 & \\
\hline 6 & 0.140 & 1027 & $1.320 \mathrm{e}+4$ & 0.7509 & N-NSGA-II \\
\hline 7 & 0.150 & 939.7 & $1.261 \mathrm{e}+4$ & 0.7503 & \\
\hline 8 & 0.151 & 928.8 & $1.261 \mathrm{e}+4$ & 0.7469 & \\
\hline 9 & 0.151 & 933.7 & $1.261 \mathrm{e}+4$ & 0.7452 & \\
\hline 10 & 0.138 & 1068 & $1.329 \mathrm{e}+4$ & 0.7426 & \\
\hline
\end{tabular}

Gantt

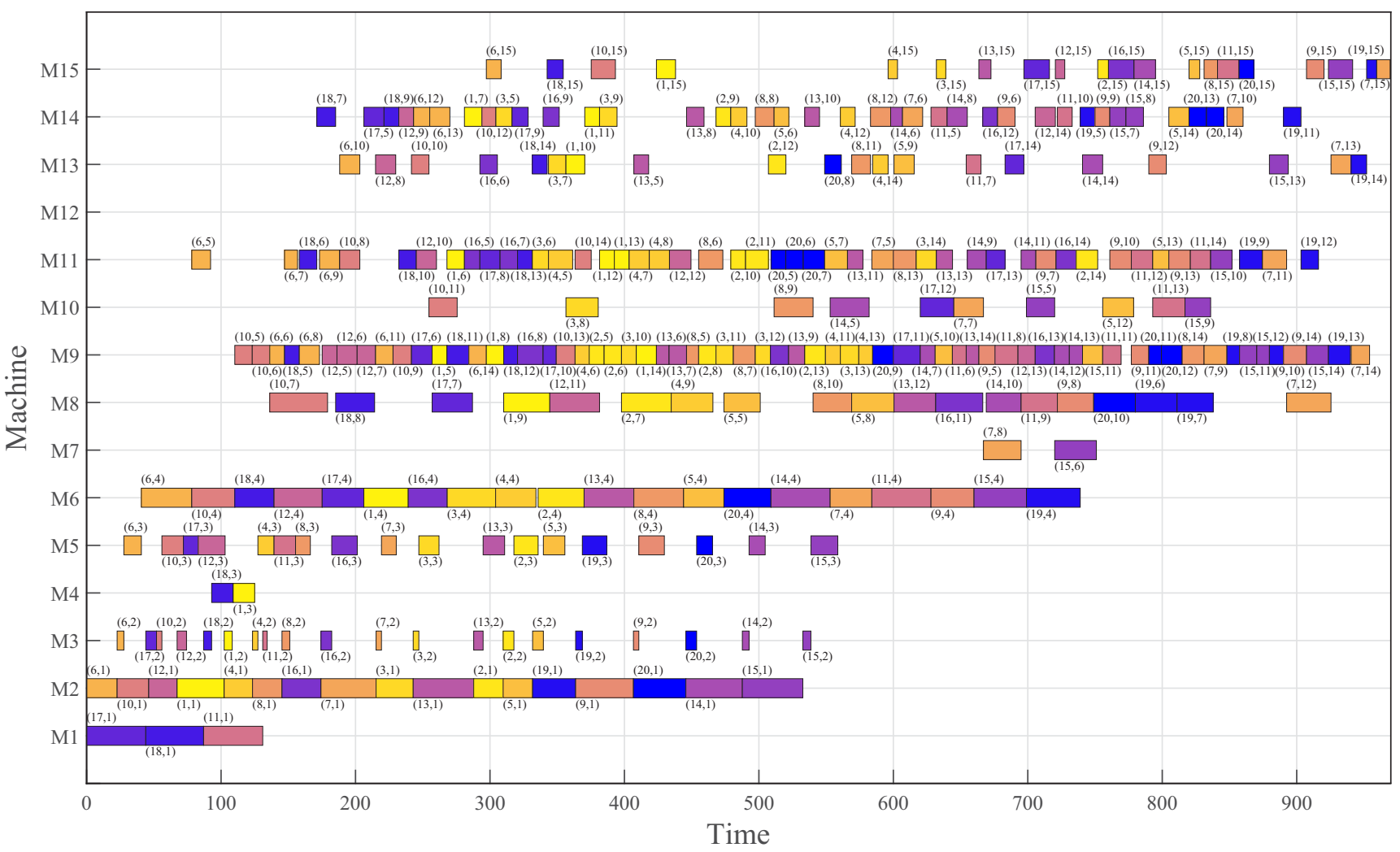

Fig. 9 The Gantt chart of the most satisfying solution for the case

the solutions obtained by N-NSGA-II is much lower than that by other algorithms under the condition of the same makespan and the minimum makespan is obtained by the proposed N-NSGA-II. In Fig. 8 (c) gives a 2D view subjected to defective rate and total energy consumption. The minimum TEC is obtained by the N-NSGA-II. Fig. 8dd) presents the results only containing the makespan and TEC. It is clear that the solutions generated by N-NSGA-II are much better than those by other algorithms.

The computational results on metrics of GD, CR and IGD are shown in Table.11 and on $c$-metric are shown in Table.12. $A, E$ and $F$ donate the N-NSGA-II, SPEA-II and NSGA-III respectively in Table.12. As shown in Table.11, the N-NSGA-II 
is significantly better than SPEA-II and NSGA-III on the metrics of GD, CR and IGD. Furthermore, as shown in Table.12, N-NSGA-II overwhelmingly dominates in pairwise comparison with SPEA-II and NSGA-III. Consequently, the superior of the proposed N-NSGA-II is validated.

Moreover, to obtain the most satisfying solution in the Pareto front, the approach in [8] is introduced to the paper. Assume that the weight vector $\boldsymbol{W}=\left(\begin{array}{lll}0.4 & 0.3 & 0.3\end{array}\right)^{T}$ regarding to the objectives of defective rate, makespan and total energy consumption respectively. By normalizing the fitness and multiplying by the $\boldsymbol{W}$, the scores of each solution are obtained. The higher score represents better quality. The top 10 scores among all solutions are listed in Table.13 and the Gantt chart of the solution with the highest score is illustrated in Fig 9 . As shown in Table.13, the top 10 scoring solutions are all obtained by N-NSGA-II, which gives further proof of the superiority of the N-NSGA-II.

\section{Conclusions and future work}

In this paper, the casting production scheduling problem(CPSP) under the shop floor environment is investigated. A set of comprehensive and practical scheduling scheme for casting production is obtained by establishing a multi-objective CPSP to minimize three objectives of defective rate, makespan and total energy consumption simultaneously. Furthermore, the NNSGA-II is proposed for solving the multi-objective CPSP. The effectiveness of the proposed neighborhood structures and novel solution generating approach of the N-NSGA-II is validated. Moreover, the overall performance of the N-NSGA-II is evaluated comprehensively via comparison experiments and the superiority of the N-NSGA-II is verified.

There are also some limitations of this work. On the aspect of problem formulation, the model accuracy can be improved by involving realistic constraints. For example, energy-intensive operations like melting and thermal treatment are often constrained to certain periods to avoid peak hours of energy consumption. Another example is the processing interval constraint which means the time interval between two certain operations is constrained according to the process requirements. Moreover, unexpected events such as immediate job insertion and machine breakdown may occur in the practical casting production workshops. On the 325 aspect of algorithm, since reinforcement learning $(\mathrm{RL})$ has made remarkable achievements in recent years, RL-based approaches will be a promising way to enhance the performance of existing heuristic algorithms. Besides, constructing the systematic rules by incorporating the knowledge of specific problems will greatly reduce the invalid search such that the performance of algorithms can be promoted.

\section{Compliance with ethical standards}

3зо Acknowledgments The authors would like to express their sincere thanks to the referees for their valuable suggestions and comments. The authors would also like to thank Prof. Qian He from Guilin University of Electronic Technology for his help and advice on the revision and proofreading of the manuscript.

Funding This work is supported by the National Key R \& D Program of China (Grant No.2018YFB1308200), National Natural Science Foundation of China (No.62073127)

335 Conflict of Interest The authors declare that they have no conflict of interest.

Ethical approval This article does not contain any studies with human participants or animals performed by any of the authors. Informed consent Informed consent was obtained from all individual participants included in the study.

\section{References}

1. Akturk, M.S., Ilhan, T.: Single cnc machine scheduling with controllable processing times to minimize total weighted tardiness. Computers \& Operations Research 38(4), 771-781 (2011)

2. Asefi, H., Jolai, F., Rabiee, M., Araghi, M.E.T.: A hybrid nsga-ii and vns for solving a bi-objective no-wait flexible flowshop scheduling problem. The International Journal of Advanced Manufacturing Technology 75, 1017-1033 (2014)

3. Atan, M.O., Akturk, M.S.: Single cnc machine scheduling with controllable processing times and multiple due dates. International Journal of Production Research 46(21), 6087-6111 (2008)

345 4. Chaudhry, I.A., Khan, A.A.: A research survey: review of flexible job shop scheduling techniques. International Transactions in Operational Research 23(3), 551-591 (2016)

5. Che, A., Lv, K., Levner, E., Kats, V.: Energy consumption minimization for single machine scheduling with bounded maximum tardiness. In: 2015 IEEE 12th International Conference on Networking, Sensing and Control, pp. 146-150 (2015)

6. Chen, X., An, Y., Zhang, Z., Li, Y.: An approximate nondominated sorting genetic algorithm to integrate optimization of production scheduling and accurate maintenance based on reliability intervals. Journal of Manufacturing Systems 54, 227-241 (2020)

7. Cheng, R., Gen, M., Tsujimura, Y.: A tutorial survey of job-shop scheduling problems using genetic algorithms-i: representation. Computers \& Industrial Engineering 30(4), 983-997 (1996)

8. Dai, M., Tang, D., Giret, A., Salido, M.A.: Multi-objective optimization for energy-efficient flexible job shop scheduling problem with transportation constraints. Robotics and Computer-integrated Manufacturing 59, 143-157 (2019)

355 9. Deb, K., Jain, H.: An evolutionary many-objective optimization algorithm using reference-point-based nondominated sorting approach, part i: Solving problems with box constraints. IEEE Transactions on Evolutionary Computation 18(4), 577-601 (2014)

10. Deb, K., Pratap, A., Agarwal, S., Meyarivan, T.: A fast and elitist multiobjective genetic algorithm: Nsga-ii. IEEE Transactions on Evolutionary Computation 6(2), 182-197 (2002)

11. Fattahi, P., Mehrabad, M.S., Jolai, F.: Mathematical modeling and heuristic approaches to flexible job shop scheduling problems. Journal of Intelligent Manufacturing 18(3), 331-342 (2007)

12. Gen, M., Lin, L.: Multiobjective evolutionary algorithm for manufacturing scheduling problems: state-of-the-art survey. Journal of Intelligent Manufacturing 25(5), 849-866 (2014) 
13. Hajej, Z., Rezg, N., Gharbi, A.: Quality issue in forecasting problem of production and maintenance policy for production unit. International Journal of Production Research 56, 6147-6163 (2018)

14. Jiang, S., Zheng, Z., Liu, M.: A preference-inspired multi-objective soft scheduling algorithm for the practical steelmaking-continuous casting production. Computers \& Industrial Engineering 115, 582-594 (2018)

15. Kayan, R.K., Akturk, M.S.: A new bounding mechanism for the cnc machine scheduling problems with controllable processing times. European Journal of Operational Research 167(3), 624-643 (2005)

16. Khouja, M., Mehrez, A.: Economic production lot size model with variable production rate and imperfect quality. Journal of the Operational Research Society 45, 1405-1417 (1994)

17. Lei, D., Li, M., Wang, L.: A two-phase meta-heuristic for multiobjective flexible job shop scheduling problem with total energy consumption threshold. IEEE Transactions on Systems, Man, and Cybernetics 49(3), 1097-1109 (2019)

18. Li, J., Pan, Q., Liang, Y.: An effective hybrid tabu search algorithm for multi-objective flexible job-shop scheduling problems. Computers \& Industrial Engineering 59(4), 647-662 (2010)

375 19. Li, J., Pan, Q., Suganthan, P.N., Chua, T.J.: A hybrid tabu search algorithm with an efficient neighborhood structure for the flexible job shop scheduling problem. The International Journal of Advanced Manufacturing Technology 52(5), 683-697 (2011)

20. Liu, G., Yang, H., Cheng, M.: A three-stage decomposition approach for energy-aware scheduling with processing-time-dependent product quality. International Journal of Production Research 55(11), 3073-3091 (2017)

21. Lu, C., Li, X., Gao, L., Liao, W., Yi, J.: An effective multi-objective discrete virus optimization algorithm for flexible job-shop scheduling problem with controllable processing times. Computers \& Industrial Engineering 104, 156-174 (2017)

22. Luo, S., Zhang, L., Fan, Y.: Energy-efficient scheduling for multi-objective flexible job shops with variable processing speeds by grey wolf optimization. Journal of Cleaner Production 234, 1365-1384 (2019)

23. Mansouri, S.A., Aktas, E., Besikci, U.: Green scheduling of a two-machine flowshop: Trade-off between makespan and energy consumption. European Journal of Operational Research 248(3), 772-788 (2016)

24. Marchi, B., Zanoni, S., Zavanella, L., Jaber, M.Y.: Supply chain models with greenhouse gases emissions, energy usage, imperfect process under different coordination decisions. International Journal of Production Economics 211, 145-153 (2019)

25. Mokhtari, H., Hasani, A.: An energy-efficient multi-objective optimization for flexible job-shop scheduling problem. Computers \& Chemical Engineering 104, 339-352 (2017)

26. Moslehi, G., Mahnam, M.: A pareto approach to multi-objective flexible job-shop scheduling problem using particle swarm optimization and local search. International Journal of Production Economics 129(1), 14-22 (2011)

27. Ouaret, S., Kenne, J., Gharbi, A.: Production and replacement policies for a deteriorating manufacturing system under random demand and quality. European Journal of Operational Research 264(2), 623-636 (2018)

28. Peng, T., Xu, X.: Energy-efficient machining systems: a critical review. The International Journal of Advanced Manufacturing Technology 72(9), $1389-1406$ (2014)

29. Piroozfard, H., Wong, K.Y., Wong, W.P.: Minimizing total carbon footprint and total late work criterion in flexible job shop scheduling by using an improved multi-objective genetic algorithm. Resources Conservation and Recycling 128, 267-283 (2018)

30. Qin, H., Fan, P., Tang, H., Huang, P., Fang, B., Pan, S.: An effective hybrid discrete grey wolf optimizer for the casting production scheduling problem with multi-objective and multi-constraint. Computers \& Industrial Engineering 128, 458 - 476 (2019)

31. Rezaeimalek, M., Tavakkolimoghaddam, R., Siadat, A., Dantan, J.: A novel model for the integrated planning of part quality inspection and preventive maintenance in a linear-deteriorating serial multi-stage manufacturing system. The International Journal of Advanced Manufacturing Technology 96, 3633-3650 (2018)

32. Shao, X., Liu, W., Liu, Q., Zhang, C.: Hybrid discrete particle swarm optimization for multi-objective flexible job-shop scheduling problem. International Journal of Advanced Manufacturing Technology 67, 2885-2901 (2013)

33. Shen, X., Han, Y., Fu, J.: Robustness measures and robust scheduling for multi-objective stochastic flexible job shop scheduling problems. Soft Computing 21, 6531-6554 (2017)

34. Soto, C., Dorronsoro, B., Fraire, H.H., Cruzreyes, L., Gomezsantillan, C., Rangel, N.: Solving the multi-objective flexible job shop scheduling problem with a novel parallel branch and bound algorithm. Swarm and evolutionary computation 53, 100632 (2020)

35. Tambe, P.P., Mohite, S., Kulkarni, M.S.: Optimisation of opportunistic maintenance of a multi-component system considering the effect of failures on quality and production schedule: A case study. The International Journal of Advanced Manufacturing Technology 69(5), $1743-1756$ (2013)

410 36. Tang, H., Chen, R., Li, Y., Peng, Z., Guo, S., Du, Y.: Flexible job-shop scheduling with tolerated time interval and limited starting time interval based on hybrid discrete pso-sa: An application from a casting workshop. Applied Soft Computing 78, 176 - 194 (2019)

37. Turkyilmaz, A., Şenvar, O., Unal, I., Bulkan, S.: A research survey: heuristic approaches for solving multi objective flexible job shop problems. Journal of Intelligent Manufacturing pp. 1-35 (2020)

38. Uruk, Z., Gultekin, H., Akturk, M.S.: Two-machine flowshop scheduling with flexible operations and controllable processing times. Computers \& Operations Research 40(2), 639-653 (2013)

39. Vilcot, G., Billaut, J.: A tabu search and a genetic algorithm for solving a bicriteria general job shop scheduling problem. European Journal of Operational Research 190(2), 398-411 (2008)

40. Wu, X., Shen, X., Li, C.: The flexible job-shop scheduling problem considering deterioration effect and energy consumption simultaneously. Computers \& Industrial Engineering 135, 1004-1024 (2019)

420 41. Xia, W., Wu, Z.: An effective hybrid optimization approach for multi-objective flexible job-shop scheduling problems. Comput. Ind. Eng. 48, 409-425 (2005)

42. Xu, W., Cao, L.: Optimal maintenance control of machine tools for energy efficient manufacturing. The International Journal of Advanced Manufacturing Technology 104(9), 3303-3311 (2019)

43. Yuan, Y., Xu, H.: Multiobjective flexible job shop scheduling using memetic algorithms. IEEE Transactions on Automation Science and Engineering 12(1), 336-353 (2015)

44. Zhang, G., Gao, L., Shi, Y.: An effective genetic algorithm for the flexible job-shop scheduling problem. Expert Systems With Applications 38(4), $3563-3573(2011)$

45. Zhang, G., Shao, X., Li, P., Gao, L.: An effective hybrid particle swarm optimization algorithm for multi-objective flexible job-shop scheduling problem. Computers \& Industrial Engineering 56(4), 1309-1318 (2009)

430 46. Zhang, G., Zhang, L., Song, X., Wang, Y., Zhou, C.: A variable neighborhood search based genetic algorithm for flexible job shop scheduling problem. Cluster Computing 22(5), 11561-11572 (2019)

47. Zitzler, E., Deb, K., Thiele, L.: Comparison of multiobjective evolutionary algorithms: Empirical results. Evolutionary Computation 8(2), 173-195 (2000)

48. Zitzler, E., Thiele, L.: Multiobjective evolutionary algorithms: a comparative case study and the strength pareto approach. IEEE Transactions on Evolutionary Computation 3(4), 257-271 (1999) 